\title{
The mv-decomposition: definition and application to the distance- 2 broadcast problem in multi-hops radio networks
}

\author{
Olivier Cogis, Benoît Darties, Sylvain Durand, Jean-Claude König, \\ and Geneviève Simonet
}

LIRMM UMR5506 - Université Montpellier II - 161, rue Ada, 34392 Montpellier Cedex 05, France

- firstname.lastname@lirmm.fr

\begin{abstract}
We present a new tool called the "mv-decomposition", and we describe some interesting algorithmic properties about it. We propose an algorithm with a complexity of $O(m)$ to build a mv-decomposition for each bipartite graph. We use this mv-decomposition to propose a solution to the distance- 2 broadcast problem in a synchronous multi-hops radio networks where adjacent transmissions are subject to interferences. More precisely, we propose two algorithms of resolution: the first one guarantees a complete distance-2 broadcast scheme using $O\left((\log n)^{2}\right)$ slots for a time complexity of $O\left(m(\log n)^{2}\right)$, while the second builds a solution with a minimal number of transmissions for a time complexity of $O(m)$.
\end{abstract}

\section{Introduction}

In a multi-hops radio network, nodes communicate with each other via multi-hops wireless links. The use of the radio medium implies some restrictions and properties: whenever a node transmits, all the nodes in its communication range may receive the transmission. Incoming messages have to be forwarded to reach nodes which are located at more than one hop from the source. Since all nodes share the same frequency channel, a collision may occurs if two or more neighbors transmit simultaneously, preventing correct reception of the message. This paper deals with the broadcast problem which refers to the sending of a message from a source node to all the other nodes of the network. We consider the simplified communication model used in $[4,5]$ : nodes send messages in synchronous slots. In each slot each node acts either as a transmitter or as a receiver. A node acting as a receiver in a given slot gets a message if and only if exactly one of its neighbors transmits in this slot. In addition, the topology of the network is assumed to be known by all the nodes. This model has been widely considered to analyze the complexity of the broadcast problem. According to this model, a valid broadcast strategy consists of finding a schedule scheme, i.e. a particular schedule of transmissions among the network nodes.

Many research have focused on producing schedule-based broadcasting schemes in known radio networks. Chlamtac and Kutten have proved that finding a scheme with a minimum number of slots is a NP-Hard problem [4]. Authors from [5] have first proposed a polynomial algorithm in $O\left(n m(\log n)^{2}\right)$ for constructing a schedule which achieves a broadcast in $O\left(D \cdot(\log n)^{2}\right)$ slots, where $D$ is the source eccentricity, 
$n$ the number of nodes and $m$ the number of links. Other results have progressively reduced this bound to $O\left(D \cdot \log n+(\log n)^{2}\right)$ in [2, 11], then $O\left(D+(\log n)^{5}\right)$ [9], to end with $O\left(D+(\log n)^{4}\right)$ [10]. In this last paper authors announce a schedule scheme for broadcasting which requires $O\left(D+(\log n)^{3}\right)$ slots when the network graph is planar. In [1], the authors present a class of 2-diameter graphs which require $\Omega\left((\log n)^{2}\right)$ slots to complete a broadcast.

The broadcast problem has also been studied under the assumption that the topology is unknown: a first scheme using $O\left(n^{11 / 6}\right)$ slots has been proposed in [6]. This bound has been decreased in multiple works $[12,7,13]$ to reach $O\left(n(\log n)^{2}\right)$ slots in [8]. Actual lower bounds for the broadcast problem without knowledge of the topology are in $\Omega(n \log n)[6,3]$.

This paper is organized as follows: in section 2 we present a new tool: the mvdecomposition, and describe some of its algorithmic properties. We use the mvdecomposition in section 3 to propose strategies with performance guarantees for the distance-2 broadcast problem: this problem is a restricted version of the broadcast problem in which the objective consists of informing nodes located at two hops away from the source node. We conclude this section by giving an algorithm which constructs a distance-2 broadcast strategy requiring $O\left((\log n)^{2}\right)$ slots. The quality of the strategy returned by our algorithm is the same as the solution proposed by [5], but the computation time complexity is improved from $O\left(n m(\log n)^{2}\right)$ to $O\left(m(\log n)^{2}\right)$.

\section{A new tool: the mv-decomposition}

This section is organized as follows: in a first step, we propose a common graph model for radio networks, and we introduce some useful definitions and notations. Then we propose a new tool, which we call the mv-decomposition, and pose some algorithmic properties. We also propose an algorithm with a complexity of $O(m)$ to compute an mv-decomposition for each bipartite graph.

\subsection{Model description and definitions}

A radio network is commonly modelized by an undirected graph $G=(V, E)$, where $V$ represents the network nodes, and $E$ contains pairs of nodes which can directly communicate. The source node is noted $s$.

Let $G=(X, Y, E)$ be a bipartite graph. A cover of a subset $Y^{\prime} \subseteq Y$ in $G$, is a subset $X^{\prime} \subseteq X$ such that $Y^{\prime} \subseteq N_{G}\left(X^{\prime}\right)$, where $N_{G}\left(X^{\prime}\right)$ is the union of neighborhoods of vertices of $X^{\prime}$ in $G$.

We say that $X^{\prime}$ is a minimal cover (for the inclusion) of $Y^{\prime}$ in $G$ when $X^{\prime}$ is a cover of $Y$, but none of its subsets is.

For a given cover $X^{\prime}$ of $Y$ in $G$, we note $m v_{G}\left(X^{\prime}\right)$ the set of neighbors of $X^{\prime}$ which are adjacent to exactly one element of $X^{\prime}$. 
Lemma 1. let $G=(X, Y, E)$ be a bipartite graph and $X^{\prime} \subseteq X$ a minimal cover of $Y^{\prime} \subseteq$ $Y$.

Then each vertex of $X^{\prime}$ has an adjacent vertex in $Y$ which is not adjacent to any other vertex of $X^{\prime}$. In other words, $m v_{g}\left(X^{\prime}\right) \geq\left|X^{\prime}\right|$.

Proof. Let $X^{\prime} \subseteq X$ be a cover of $Y^{\prime} \subseteq Y$ in $G_{B}$, and $x$ be a vertex of $X^{\prime}$. If each neighbor of $x$ in $Y$ is also adjacent to another vertex in $X^{\prime}$, then $X^{\prime}-\{x\}$ is still a cover of $Y^{\prime}$.

Let $G=(X, Y, E)$ be a bipartite graph such that $X$ covers $Y$. We say that a collection $\left(X_{i}\right)_{i \in I}$ of subsets of $X$ saturates $Y$ in $G_{B}$ when $Y=\bigcup_{i \in I} m v_{G}\left(X_{i}\right)$. Then the saturation cost of $G$ is the minimal cardinal of a collection of subsets of $X$ which saturates $Y$ in $G$. We note it $\sigma(G)$.

\subsection{The mv-decomposition: definition and properties}

In the following sections, let us define $X_{0}=X$ and $Y_{0}=Y$. A mv-decomposition of a bipartite graph $G$ consists of the data of an integer $K$, a collection $\left(X_{i}\right)_{1 \leq i \leq K}$ of $K$ subsets of $X$ which saturates $Y$ in $G$, and two others collections $\left(Y_{i}\right)_{1 \leq i \leq K}$ and $\left(Z_{i}\right)_{1 \leq i \leq K}$, such that for each $i$ with $X_{i} \neq \emptyset$ we have :

- $X_{i+1} \subseteq X_{i}$ is a minimal cover of $Y_{i}$,

- $Z_{i}$ is defined such that the subgraph of $G$ induced by $X_{i} \cup Z_{i}$ is a perfect matching: each vertex has degree 1 ,

$-Y_{i+1}=Y_{i}-Z_{i+1}$.

The depth of an mv-decomposition is the smallest value $K$, for which $Y_{K}=\emptyset$. Let us note that, for any collection $\left(X_{i}\right)_{1 \leq i \leq K}$ which saturates $Y$ in $G$, one can deduce an mv-decomposition of depth $K$, by computing the sets $Y_{i}$ and $Z_{i}$ from the knowledge of $X_{i}$. That is why, in the following, an mv-decompostion is sometime described as the collection $\left(X_{i}\right)_{1 \leq i \leq K}$.

Property 1 Let $G=(X, Y, E)$ be a bipartite graph such that $X$ covers $Y$. Then for each mv-decomposition we have:

1. $\left\{X_{i}\right\}_{0 \leq i \leq K}$ et $\left\{Y_{i}\right\}_{0 \leq i \leq K}$ are two sequences such that $X_{i} \subseteq X_{i-1}$ and $Y_{i} \subseteq Y_{i-1}$, with $X_{K} \neq \emptyset$ and $Y_{K}=\emptyset$. In addition $X_{i}$ covers $Y_{i}$ for $0 \leq i \leq K$.

2. $\left\{Z_{j}\right\}_{i \leq j \leq K}$ is a partition of $Y_{i-1}$. In particular $\left\{Z_{i}\right\}_{1 \leq i \leq K}$ is a partition of $Y$.

3. For each $i$ such that $1 \leq i \leq K$, we have $\left|Z_{i}\right|=\left|X_{i}\right| \neq \emptyset$, and $Z_{i} \subseteq m v_{G}\left(X_{i}\right)$.

4. For each $i$ such that $1 \leq i \leq K$, each vertex $x$ of $X_{i}$ has, for each $j$ such that $1 \leq j \leq i$, exactly one neighbor in $Z_{j}$ which is not adjacent to any other vertex of $X_{i}$.

Proof. Let us consider $X_{i} \subseteq X$ and $Y_{i} \subseteq Y, Y_{i} \neq \emptyset$, such that $X_{i}$ covers $Y_{i}$ (true for $i=0$ ). Then $Y_{i}$ has some minimal cover $X_{i+1} \subseteq X_{i}$. Lemma 1 allows to affirm that for each $X_{i+1} \neq \emptyset, Z_{i+1}$ is defined and not empty, and then that $Y_{i+1}$ is strictly included in $Y_{i}$. This also guarantees that $X_{i+1}$ is a cover of $Y_{i+1}$. This proves points (1), (2) and (3). 


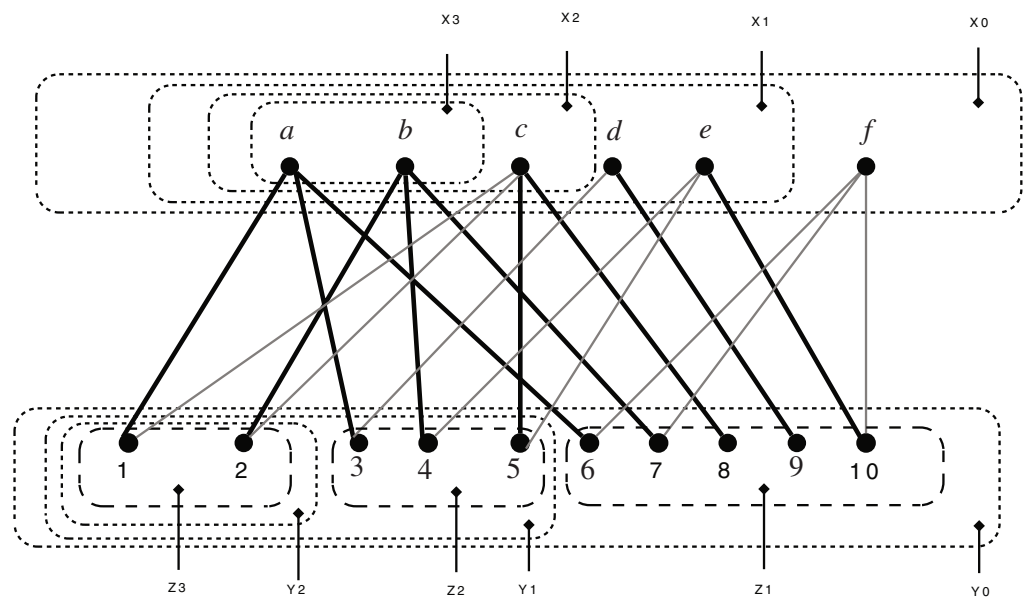

Fig. 1 an mv-decomposition of a bipartite graph

For each $i$ and $j$ such that $1 \leq j \leq i \leq K$, we have $X_{i} \subseteq X_{j}$. Then any vertex $x$ of $X_{i}$ is also a vertex of $X_{j}$. Since the subgraph of $G$ induced by vertices $X_{j} \cup Z_{j}$ is a perfect matching, then there exists a vertex $z_{j} \in Z_{j}$ adjacent to $x$ but not with any other vertex of $X_{j}$.

For any mv-decomposition of $G$ with a depth $K$, we have $K \leq \Delta_{G}(X)$, where $\Delta_{G}(X)$ is the maximum degree of a node of $X$ in $G$.

Property 2 Let $G=(X, Y, E)$ be a bipartite graph such that $X$ covers $Y$. Then for any $m v$-decomposition of $G$ with a depth $K$, we have:

$$
K \leq \Delta_{G}(X)
$$

Where $\Delta_{G}(X)$ is the maximum degree of a vertex of $X$ in $G$.

Proof. According to points 2 and 4 of property 1 , we have $d_{G}(x) \geq K$. This allows us to conclude.

We propose the algorithm "mv-decomposition" which computes an mvdecomposition from a given bipartite graph $G=(X, Y, E)$. 


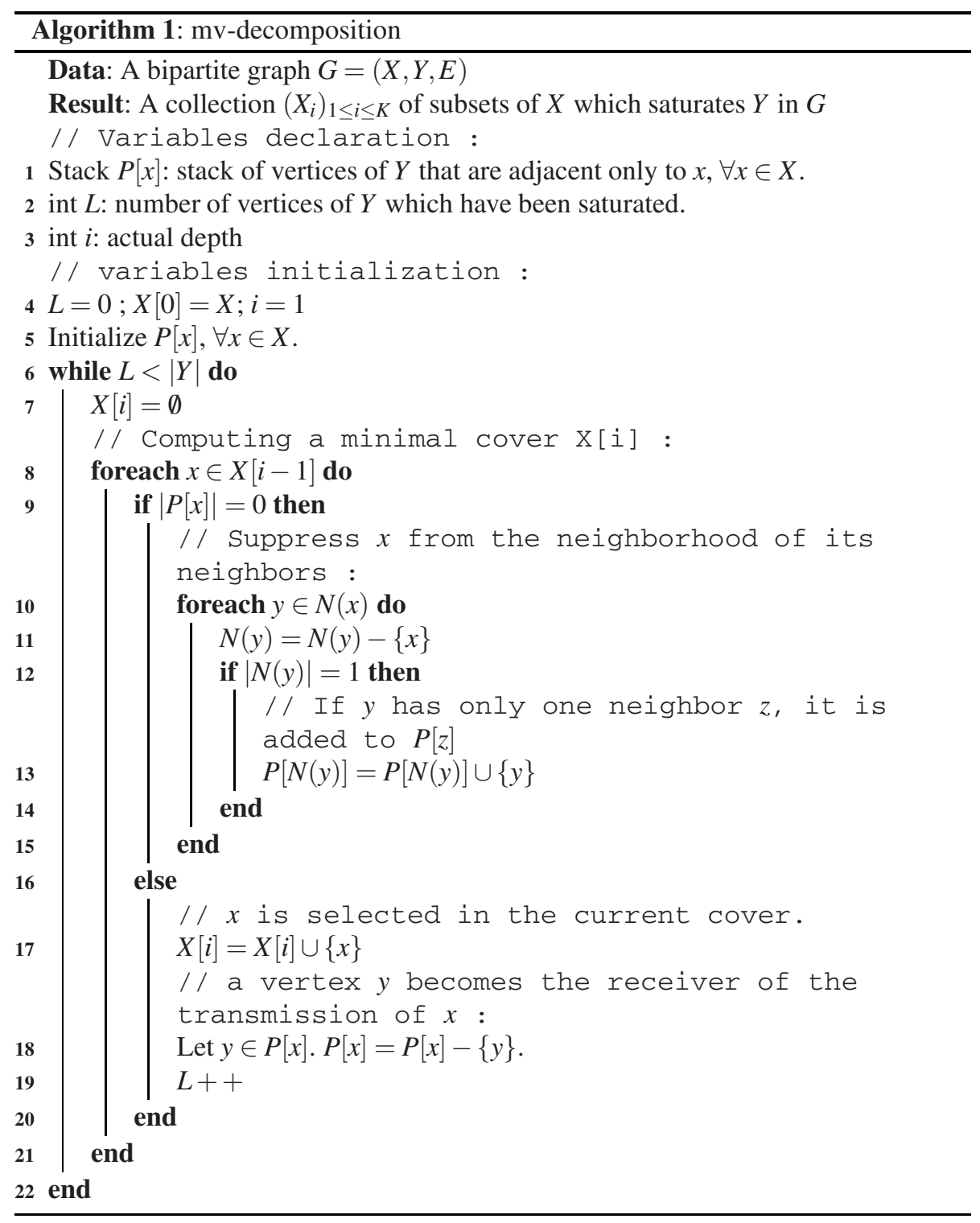

Theorem 1 The algorithm $m v$-decomposition has a complexity of $O(m)$.

Proof. The initialization phase (line 5) runs in $O(m)$ and consists of filling the stacks $P[x], \forall x \in X$.

Thereafter, for a given $x \in X$ :

- The part of code between lines 9 and 16 is executed at most once, and consists of suppressing the vertex $x$ from $G$.

- The part of code between lines 17 and 21 is executed at most $d_{G}(x)$ times. 
The part of code between lines 9 and 15 has a complexity of $O\left(d_{G}(x)\right)$ (by using advanced implementation techniques). The part of code between lines 17 and 21 has a complexity of $O(1)$.

It is concluded that the overall complexity of the algorithm is of the order of :

$$
O\left(\sum_{x \in X} d_{G}(x)\right)=O(m)
$$

\section{Using the mv-decomposition to solve the distance-2 broadcast problem}

We employ the mv-decomposition to define solutions with performance guarantees for the distance- 2 broadcast problem in multi-hops synchronous radio networks. This problem is a particular case of the broadcast-problem and can be described as follows: let us consider a single source broadcast problem. After the first slot is completed, all the nodes which are adjacent to the source node have a knowledge of the broadcasted information. Their transmissions must be scheduled in order to inform all the nodes that are two hops away from the source. A recursive approach of this process, depending on the distance of nodes from the source, allows to broadcast the message on the whole network.

The data can be restricted to a bipartite graph $G=\left(X, Y, E_{B}\right)$ where $X$ and $Y$ respectively denotes the set of vertices at distance 1 and 2 of $s$ in $G$, and $E$ the set of possible direct communications: $E_{B}=\{\{x, y\} \mid x \in X, y \in Y,\{x, y\} \in E\}$. We say that finding a distance- 2 broadcast strategy consists of broadcasting a single message from nodes of $X$ to nodes of $Y$. In a synchronous model, two important criterias are the number of required slots, and the number of realized transmissions.

In the first sub-section, we use the mv-decomposition to propose a distance- 2 broadcast strategy with a minimal (not minimum, which is an NP-hard problem) number of transmissions, and a number of slots bounded by the maximum degree of the graph. In the second sub-section, we propose an algorithm to compute a distance- 2 broadcast strategy with $\left.O(\log n)^{2}\right)$ slots, for a time complexity of $O\left(m(\log n)^{2}\right)$.

\subsection{Minimizing both the number of slots and the number of transmissions}

Let $I$ be an instance of the distance-2 broadcast problem composed of a bipartite graph $G=(X, Y, E)$ such that $X$ covers $Y$.

The following theorem establishes a link between a cover of $Y$ in a bipartite graph, and the number of required transmissions for the distance- 2 broadcast problem on the same graph. 
Theorem 2 Let $G=(X, Y, E)$ be a bipartite graph such that $X$ covers $Y$. Then we have:

1. If $C$ is a minimal cover of $Y$, then there exists a broadcast strategy from $X$ to $Y$ with a minimal number of transmissions equal to the cardinality of $C$.

2. Finding a broadcast strategy with a minimum number of transmissions is tantamount to finding a minimum cover.

Proof. Let $C \subseteq X$ be a minimal cover of $Y$ of cardinality $k$, with $C=\left\{c_{i}\right\}_{1 \leq i \leq k}$. Let us consider the following strategy : during each slot exactly one node of $C$ is transmitting. All the nodes of $C$ have transmitted the information after $k$ slots, and no interference has occurred. Thus all the nodes of $Y$ have successfully received the information, and we infer points 1 and 2 . The number of transmissions is clearly equal to the cardinality of the cover $C$. Let us note that as $C$ is minimal, each element of $C$ has to transmit at least once. Q.E.D.

Let us consider a collection $\left(X_{i}\right)_{1 \leq i \leq K}$ of subsets of $X$ resulting from the mvdecomposition of $G$. From this mv-decomposition we can propose a distance-2 broadcast strategy $S_{1}$ : At slot $i$, all the nodes of $X_{K+1-i}$ are transmitting the message. Since the collection $\left(X_{i}\right)_{1 \leq i \leq K}$ saturates $Y$, each node of $Y$ can receive the information. The number of transmissions is equal to $\sum_{i=1}^{K}\left|X_{i}\right|$. This number is not minimal, since $X_{1}$ is already a minimal cover of $Y$.

We define a second strategy $S_{2}$ as follows :

- During the first slot, all the nodes of $X_{K}$ transmit the message.

- During the slot $i$ with $2 \leq i \leq K$, all the nodes of $X_{K+1-i}-X_{K+2-i}$ transmit the message.

This second strategy differs from the previous one in the fact that when a node transmits at slot $i$, it does not transmit anymore. We propose the following property :

Property 3 The strategy $S_{2}$ produces a complete broadcast from $X$ to $Y$.

sketch of proof: We recall that $X_{i+1} \subseteq X_{i}$ for all $i$ such that $1 \leq i \leq k-1$. Each node of $X_{1}$ transmits exactly once. The validity of this strategy can be deduced if we compare it with $S_{1}$.

The number of used slots by strategy $S_{2}$ is $K$. Its cost in number of transmissions is equal to:

$$
\left|X_{K}\right|+\sum_{i=2}^{K}\left|X_{K+1-i}-X_{K+2-i}\right|=\left|X_{1}\right|
$$

If the set $X_{1}$ is a minimal cover of $Y$, then we obtain a valid broadcast strategy (all the nodes will receive the information), where the number of transmissions is minimal, in accordance with Theorem 2.

With both strategies $S_{1}$ and $S_{2}$, the number of used slots is less or equal than $\Delta_{G}(X)$, in agreement with property 2 . We show in the following sub-section that we can obtain a strategy with a better cost in term of number of slots. 


\subsection{Minimizing the number of slots}

In this sub-section, we propose another strategy to solve the distance- 2 broadcast problem. The objective is here to minimize the number of used slots, regardless the number of effective transmissions.

Our approach consists of showing that one can ensure that enough number of nodes of $Y$ could receive the message in exactly one slot. By generalizing this property, we obtain a valid broadcast strategy, and we evaluate its cost in number of slots. First we establish some properties concerning the receptivity, ie the maximum number of nodes that can receive a transmission correctly in one slot.

Property 4 Let $G=(X, Y, E)$ be a bipartite graph such that $X$ covers $Y$. Then the receptivity $\rho(G)$ satisfies the following inequation:

$$
\max _{X^{\prime} \subseteq X}\left|m v_{G}\left(X^{\prime}\right)\right|=\rho(G) \geq \max \left(\Delta_{G}(X), \frac{|Y|}{\Delta_{G}(X)}\right)
$$

Proof. Let $x$ be a vertex of $X$ having degree $\Delta_{G}(X)$. Then $\left|m v_{G}(\{x\})\right|=\Delta_{G}(X)$. The inequality $\rho(G) \geq \Delta_{G}(X)$ is deduced from the definition of $\rho(G)$.

Let $X^{\prime} \subseteq X$ be a minimal cover of $Y$ in $G$. While $Y=\bigcup_{x \in X^{\prime}} N_{G}(x)$, then we have :

$$
|Y| \leq \sum_{x \in X^{\prime}}\left|N_{G}(x)\right|=\sum_{x \in X^{\prime}} d_{G}(x) \leq\left|X^{\prime}\right| \cdot \Delta_{G}(X) \leq m v_{G}\left(X^{\prime}\right) \cdot \Delta_{G}(X)
$$

The second inequality $\rho(G) \geq \frac{|Y|}{\Delta_{G}(X)}$ is deduced again from the definition of $\rho(G)$.

As an immediate corollary of property 4 , we have :

$$
\rho(G) \geq \sqrt{|Y|}
$$

In fact, we are going to improve this bound to show that :

$$
\rho(G) \geq \frac{|Y|}{1+\ln |Y|}
$$

Property 5 Let $G=(X, Y, E)$ be a bipartite graph such that $X$ covers $Y$. Then for each $m v$-decomposition for $G$, we have :

$$
\forall i|1 \leq i \leq K,| m v_{G}\left(X_{i}\right)|\geq i \times| X_{i} \mid
$$

Proof. According to point 4 of property 1, each node $x$ of $X_{i}$ has in each $Z_{j}$, with $1 \leq j \leq i$, a neighbor which is not adjacent to any other vertex of $X_{i}$. According to point 2 of the same property, these $i$ neighbors are pairwise distinct.

Theorem 3 Let $G=(X, Y, E)$ be a bipartite graph such that $X$ covers $Y$. Then for each $m v$-decomposition of $G$, we have : 


$$
\begin{gathered}
\rho(G) \geq \max _{1 \leq i \leq K}\left|m v_{G}\left(X_{i}\right)\right| \geq \frac{|Y|}{H_{K}} \\
\sigma(G) \leq K
\end{gathered}
$$

where $H_{n}$ is the harmonic number $H_{n}=1+\frac{1}{2}+\frac{1}{3}+\cdots+\frac{1}{n}$.

Proof. The first inequality of (3) stems from the definition of $\rho(G)$. The second is deduced from the followings:

$$
\begin{aligned}
|Y| & =\sum_{i=1}^{K}\left|Z_{i}\right| \\
& =\sum_{i=1}^{K}\left|X_{i}\right| \\
& \leq \sum_{i=1}^{K} \frac{\left|m v_{G}\left(X_{i}\right)\right|}{i} \\
& \leq \sum_{i=1}^{K} \frac{\max _{1 \leq i \leq K}\left|m v_{G}\left(X_{i}\right)\right|}{i}=\max _{1 \leq i \leq K}\left|m v_{G}\left(X_{i}\right)\right| \times H_{K}
\end{aligned}
$$

Now we prove the inequality 4. Let $y$ be any vertex of $Y$. According to point 2 of property $1, y \in Z_{i}$ for one $i$ such that $1 \leq i \leq K$. According to point 3 of the same property, $y \in m v_{G}\left(X_{i}\right)$. Then $Y=\bigcup_{i=1}^{K} m v_{G}\left(X_{i}\right)$, and allows us to conclude.

Theorem 4 Let $G=(X, Y, E)$ be a bipartite graph such that $X$ covers $Y$. Then :

$$
\begin{gathered}
\rho(G) \geq \frac{|Y|}{1+\ln \Delta_{G}(X)} \\
\sigma(G) \leq \Delta_{G}(X)
\end{gathered}
$$

(Let us remind that $\sigma(G)$ is the saturation cost of $G$, i.e. the minimal cardinality of a collection of subsets of $X$ which saturates $Y$ in $G)$. We note it $\sigma(G)$.

Proof. Can be deduced from theorem 3 and property 2, bearing in mind that the harmonic number $H_{n}$ is an increasing function of $n$ which satisfies $H_{n} \leq 1+\ln n$.

We propose the following algorithm : 


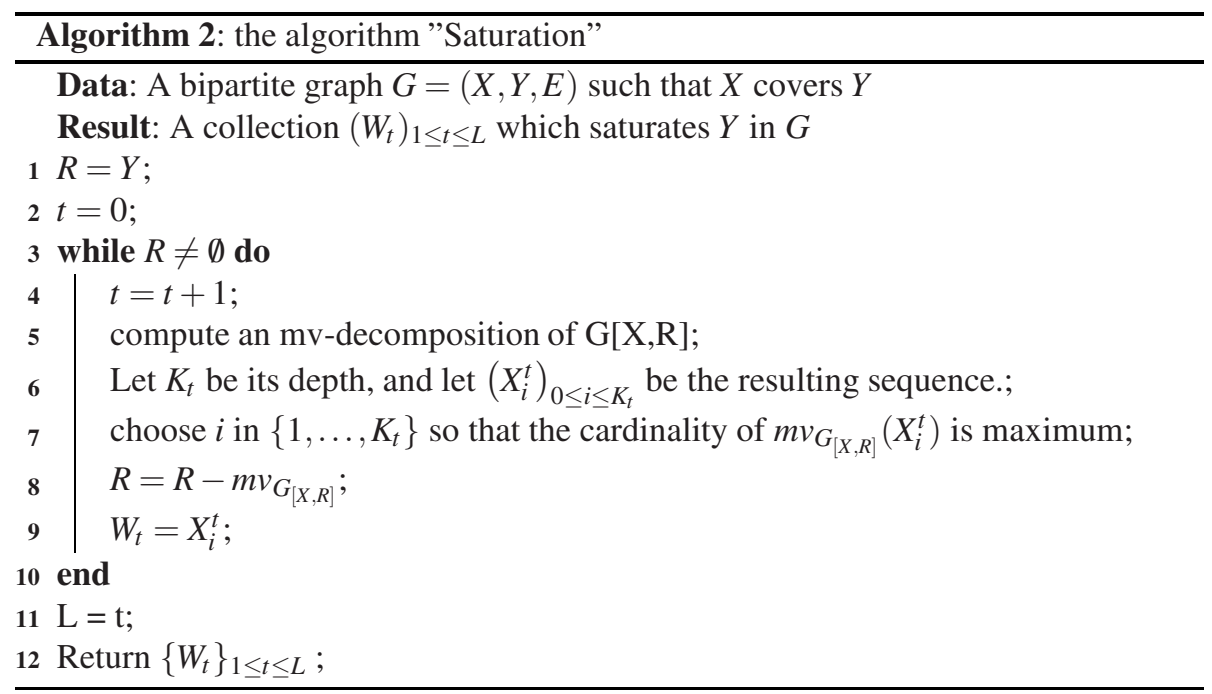

Clearly, $\left\{W_{t}\right\}_{1 \leq t \leq L}$ is a collection of subsets of $X$ and saturates $Y$.

A valid broadcast strategy can be logically deduced from $\left\{W_{t}\right\}_{1 \leq t \leq L}$, if the vertices of $W_{i}$ emit at slot $i$. The number of slots is the cardinality of $\left\{W_{t}\right\}_{1 \leq t \leq L}$, ie the number of iterations of the algorithm.

Theorem 5 The algorithm "'Saturation" runs in $O\left((\ln |Y|)^{2}\right)$ iterations. In other words, a broadcast strategy constructed from the collection $\left(W_{t}\right)_{1 \leq t \leq L}$ requires $O\left((\ln |Y|)^{2}\right)$ slots.

Proof. In agreement with property $2, K_{t} \leq \Delta_{G_{[X, R]}} \leq|R|$. During one iteration we have, in accordance with theorem 3 :

$$
\left|m v_{G_{[X, R]}}\left(X_{i}^{t}\right)\right| \geq \frac{|R|}{H_{k}} \geq \frac{|R|}{1+\ln |R|}
$$

Let us note $u_{n}$ the cardinality of the set $R$ after the $n^{\text {th }}$ iteration. Then we have :

$$
\begin{aligned}
u_{0} & =|Y| \\
u_{n+1} & \leq u_{n}\left(1-\frac{1}{1+\ln u_{n}}\right), 0 \leq n \leq L \\
u_{L} & =0
\end{aligned}
$$

Let $\left(v_{n}\right)_{n \in N}$ be the geometric sequence defined as:

$$
v_{n}=|Y|\left(1-\frac{1}{1+\ln |Y|}\right)^{n}
$$

We have : 


$$
v_{\theta\left((\ln |Y|)^{2}\right)}=0
$$

Indeed :

$$
\begin{aligned}
v_{n}<1 & \Leftrightarrow \ln v_{n}<0 \\
& \Leftrightarrow \ln |Y|+n \ln \left(1-\frac{1}{1+\ln |Y|}\right)<0
\end{aligned}
$$

While $\ln (1+x) \leq x$, in order that $v_{n}<1$, it requires that $\ln |Y|-\frac{n}{1+\ln |Y|}<0$, soit $n>\ln |Y| \times(1+\ln |Y|)$.

Clearly we have $v_{n} \geq u_{n}, \forall n$ and then $L \leq \ln |Y| \times(1+\ln |Y|)$.

Theorem 6 The algorithm "Saturation" has a time complexity of $O\left(m \times(\ln |Y|)^{2}\right)$.

Proof. During each iteration, the algorithm computes an mv-decomposition. In agreement with theorem 1 , any mv-decomposition can be computed in $O(m)$. Let us recall that the number of iterations of this algorithm is in $O\left((\ln |Y|)^{2}\right)$, in accordance with theorem 5 .

Thus, we have proposed an algorithm to compute a strategy using $O\left((\log n)^{2}\right)$ slots. The quality of the solution returned by our algorithm is the same as the algorithm of [5], but we have improved the complexity from $O\left(m n(\log n)^{2}\right)$ to $O\left(m(\log n)^{2}\right)$.

\section{Conclusion}

We have proposed the mv-decomposition as a new theoretical tool with interesting algorithmic properties. These properties have been used to develop different algorithms for the distance-2 broadcast problem in multi-hops synchronous radio networks. The mv-decomposition allows to create broadcast solutions where the number of transmissions is minimal, ensuring a number of slots below the maximum degree of the graph. The algorithm which computes this solution has a complexity of $O(m)$.

We have also proposed an algorithm which builds a distance- 2 broadcast strategy of $O\left((\ln |Y|)^{2}\right)$ slots for a time complexity $O\left(m(\log n)^{2}\right)$. This improves the result of [5] which announces a broadcast strategy with the same number of slots for a time complexity $O\left(m n(\log n)^{2}\right)$. An interesting perspective would be to adapt the mvdecomposition for the distance-3 broadcast problem, by including a weight function on the elements of $Y$, and to generalize this approach for the broadcast problem on arbitrary graphs.

\section{References}

1. N. Alon, A. Bar-Noy, N. Linial, and D. Peleg. A lower bound for radio broadcast. J. Comput. Syst. Sci., 43(2):290-298, 1991. 
2. R. Bar-Yehuda, O. Goldreich, and A. Itai. On the time-complexity of broadcast in multi-hop radio networks: An exponential gap between determinism and randomization. J. Comput. Syst. Sci., 45(1):104-126, 1992.

3. D. Bruschi and M. Del Pinto. Lower bounds for the broadcast problem in mobile radio networks. Distrib. Comput., 10(3):129-135, 1997.

4. I. Chlamtac and S. Kutten. On broadcasting in radio networks - Problem analysis and protocol design. IEEE Transactions on Communications, 33:1240-1246, December 1985.

5. I. Chlamtac and $\mathrm{O}$. Weinstein. The wave expansion approach to broadcasting in multihop radio network. IEEE Transaction Communication, (39):426-433, 1991.

6. B. Chlebus, L. Gąsieniec, A. Gibbons, A. Pelc, and W. Rytter. Deterministic broadcasting in unknown radio networks. In SODA '00: Proceedings of the eleventh annual ACM-SIAM symposium on Discrete algorithms, pages 861-870, Philadelphia, PA, USA, 2000. Society for Industrial and Applied Mathematics.

7. B. Chlebus, L. Gạsieniec, A. Östlin, and J.M. Robson. Deterministic radio broadcasting. In ICALP '00: Proceedings of the 27th International Colloquium on Automata, Languages and Programming, pages 717-728, London, UK, 2000. Springer-Verlag.

8. M. Chrobak, L. Gasieniec, and W. Rytter. Fast broadcasting and gossiping in radio networks. $J$. Algorithms, 43(2):177-189, 2002.

9. I. Gaber and Y. Mansour. Broadcast in radio networks. In SODA '95: Proceedings of the sixth annual ACM-SIAM symposium on Discrete algorithms, pages 577-585, Philadelphia, PA, USA, 1995. Society for Industrial and Applied Mathematics.

10. G. Kortsarz and M. Elkin. An improved algorithm for radio broadcast (submitted), 2005.

11. D. R. Kowalski and A. Pelc. Centralized deterministic broadcasting in undirected multi-hop radio networks. In APPROX-RANDOM, pages 171-182, 2004.

12. G. De Marco and A. Pelc. Faster broadcasting in unknown radio networks. Inf. Process. Lett., 79(2):53-56, 2001.

13. D. Peleg. Deterministic radio broadcast with no topological knowledge, 2000. 